6. Correction for hydrostatic force. Since gravity has been neglected above, it is interesting to have a rough estimate of the effect of gravity on the pressure exerted by a liquid on a missile moving through it with wetted area $W^{\prime}$ trailed by a cavity (Fig. 2). We suppose the liquid incompressible, and bounded by $W^{\prime}$, container walls $W^{\prime \prime}$, and a free surface $S$. The additional instantaneous acceleration $\mathbf{b}$ due to a vertical gravity field with intensity $g$ satisfies $\mathbf{b}=g \nabla B$, where $\nabla^{2} B=0, B=y$ (depth coordinate) on $S$, and $\partial B / \partial n=0$ on $W^{\prime}+W^{\prime \prime}=W$; the associated hydrostatic pressure is $\rho g(y-B)$.

For given boundary configurations $S$ and $W$, the resulting "hydrostatic acceleration potential" $g B$ can be most easily estimated using an electrolytic tank, and the results interpreted in terms of the dimensionless cavity buoyancy coefficient

$$
C_{H}=\frac{\text { hydrostatic bouyancy force }}{\rho g \times \text { mean depth } \times \text { horizontal projection of } W^{\prime}}
$$

In this way, $C_{H}$ was estimated ${ }^{7}$ for three two-dimensional cavity flows, having profiles similar to that of Fig. 2. The cavity buoyancy coefficients averaged about 25 per cent.

\title{
References
}

1. G. Birkhoff, Hydrodynamics: a study in logic, fact, and similitude, Princeton, 1950, esp. Chap. V.

2. L. M. Milne-Thomson, Theoretical hydrodynamics, Macmillan, 1939.

3. M. Schiffer and G. Szegö, Trans. Am. Math. Soc. 67, 130-205 (1949).

4. L. I. Sedov, CAHI Report 187 (1934), Moscow.

5. M. Shiffman and D. C. Spencer, Quar. Appl. Math. 5, 270-88 (1947), and The force of impact on a lsphere striking a water surface, AMP Report 42.1R, Feb. 1945.

6. Th. von Kármán, NACA Techn. Rep. 321 (1929).

7. G. Birkhoff and R. Isaacs, "Transient cavities in air-water entry", Navord Report 1940, January, 1951.

\section{ON THE NON-UNIQUENESS OF PERIODIC SOLUTIONS FOR AN ASYMMETRIC LIENARD EQUATION*}

By G. F. D. DUFF AND N. LEVINSON (Massachusetts Institute of Technology)

The following result has been stated by H. Serbin [5, Theorem II]. Let $f(x), g(x)$ be continuous for $-\infty<x<\infty$, and let

$$
\begin{aligned}
& f(x)<0, \quad-x_{1}^{\prime}<x<x_{1}, \\
& f(x)>0, \quad x<-x_{1}^{\prime}, \quad x_{1}<x,
\end{aligned}
$$

where $x_{1}^{\prime}>0$ and $x_{1}>0$. Let

$$
\begin{gathered}
\int_{0}^{\infty} f(x) d x>0 \\
x g(x)>0, \quad x \neq 0
\end{gathered}
$$

${ }^{*}$ Received Aug. 20, 1951. This paper was written in the course of research sponsored in part by the Office of Naval Research. 
and let either $\int_{0}^{\infty} f(x) d x$ or $\int_{0}^{\infty} g(x) d x$ diverge. Then the equation

$$
\ddot{x}+f(x) \dot{x}+g(x)=0, \quad \dot{x}=d x / d t,
$$

has a unique periodic solution.

In case $g(x) \equiv x$ and $f(x)$ is even (1.4) has been treated by Liénard [3]. In case $f(x)$ is even and $g(x)$ is an odd function (1.4) has been treated by Levinson and Smith [2]. Here an example will be given with $g(x) \equiv x$ and with $f(x)$ meeting the requirements (1.0), (1.1) and (1.2) where at least three periodic solutions for (1.4) exist thus showing that the result of Serbin is incorrect as stated.

We note that if $f(x)$ satisfies the required conditions so does $\epsilon f(x)$ for any $\epsilon>0$. The equation to be considered here is

$$
\ddot{x}+\epsilon f(x) \dot{x}+x=0
$$

where $f(x)$ is a polynomial. If $x=r \cos \theta$ and $x^{\circ}=-r \sin \theta$ then (1.5) yields $r^{\circ}=\epsilon r$ $f(r \cos \theta) \sin ^{2} \theta, \theta^{\cdot}=1+\epsilon f(r \cos \theta) \sin \theta \cos \theta$ from which follows

$$
\frac{d r}{d \theta}=\frac{\epsilon r f(r \cos \theta) \sin ^{2} \theta}{1+\epsilon f(r \cos \theta) \sin \theta \cos \theta} .
$$

For each solution of (1.6) $r=r(\theta)$ periodic of period $2 \pi$ there corresponds a periodic solution of (1.5).

Since with $\epsilon=0$ the solutions of (1.6) $r=$ const., are bounded and since the right side of (1.6) is analytic in $r, \theta$ and $\epsilon$ it follows from the successive approximations existence theorem, as indicated for example in Lefschetz [1, Chap. II] that the solutions of (1.6) with $r=\rho$ for $\theta=0$ are given by $r=H(\theta, \rho, \epsilon)$ where $H$ is analytic in $(\theta, \rho, \epsilon)$ for any finite range such as $0 \leq \theta \leq 2 \pi, \frac{1}{2} \leq \rho \leq 5$, and $|\epsilon|$ sufficiently small. Thus $H$ can be expanded in powers of $\epsilon$ yielding

$$
r=H_{0}(\theta, \rho)+\epsilon H_{1}(\theta, \rho)+\epsilon^{2} \gamma(\theta, \rho, \epsilon)
$$

where $|\gamma|$ is uniformly bounded for the above range of $\theta$ and $\rho$ for $|\epsilon|$ small. Using $r=H$ in (1.6) it follows, equating powers of $\epsilon$ on each side of the equation, that $H_{0}(\theta, \rho)=$ $\rho$ and

$$
H_{1}(\theta, \rho)=\int_{0}^{\theta} \rho f(\rho \cos \sigma) \sin ^{2} \sigma d \sigma .
$$

If

$$
F(\rho)=\int_{0}^{2 \pi} \rho f(\rho \cos \sigma) \sin ^{2} \sigma d \sigma
$$

then

$$
H(2 \pi, \rho, \epsilon)-\rho=\epsilon F(\rho)+\epsilon^{2} \gamma(2 \pi, \rho, \epsilon) .
$$

If $H(2 \pi, \rho, \epsilon)-\rho$ vanishes for some $\rho$ then as already stated (1.5) has a periodic solution passing through the point $x=\rho, \dot{x}=0$ in the phase plane of the variables $(x, \dot{x})$. Let $\rho_{0}>0$ and let $F\left(\rho_{0}\right)=0$ and let $F(\rho)$ change sign at $\rho=\rho_{0}$. Then for a given small $\delta>0$, it follows from (1.8) that $H(2 \pi, \rho, \epsilon)-\rho$ has opposite signs for $\rho=\rho_{0}-\delta$ and $\rho=\rho_{0}+\delta$ providing $\epsilon$ is small enough. Since $H(2 \pi, \rho, \epsilon)-\rho$ is continuous in $\rho$ this implies that $H(2 \pi, \rho, \epsilon)-\rho$ vanishes for some $\rho$ between $\rho_{0}-\delta$ and $\rho_{0}+\delta$.

It follows therefore that if it is shown that $F(\rho)$ in (1.7) has 3 positive zeros and 
changes sign at each zero then for small $\epsilon(1.5)$ has at least three periodic solutions as stated. [4]

Let

$$
f(x)=A_{3} x^{6}-A_{2} x^{4}+A_{1} x^{2}-A_{0}-C x
$$

where $A_{i}$ and $C$ are constants. Let

$$
I_{k}=\int_{0}^{2 \pi} \sin ^{2} \sigma \cos ^{2 k} \sigma d \sigma, \quad k=0,1,2,3 .
$$

Then $I_{k}>0$ and from (1.7) it follows that

$$
F(\rho)=A_{3} I_{3} \rho^{7}-A_{2} I_{2} \rho^{5}+A_{1} I_{1} \rho^{3}-A_{0} I_{0} \rho .
$$

Let $A_{3} I_{3}=1, A_{2} I_{2}=14, A_{1} I_{1}=49$ and $A_{0} I_{0}=36$. Then all the $A_{i}$ are positive and

$$
F(\rho)=\rho\left(\rho^{2}-1\right)\left(\rho^{2}-4\right)\left(\rho^{2}-9\right) .
$$

Thus $F(\rho)$ vanishes at $\rho=1,2$, and 3 and changes sign at each of these zeros which proves that (1.5) has at least three periodic solutions for small $\epsilon$.

Since (1.2) is assured by $A_{3}>0$ in (1.9) it remains only to show that $f(x)$ satisfies (1.0) and (1.1). Since the $A_{i}$ are already fixed and since $A_{3}>0$ and $A_{0}>0$ it follows easily that if $C$ is large enough then $C x=A_{3} x^{6}-A_{2} x^{4}+A_{1} x^{2}-A_{0}$ has only one negative root, which is near $x=0$ and will be designated by $-x_{1}^{\prime}$, and only one positive root, which is large and will be designated by $x_{1}$. Clearly $f(x)$ now satisfies (1.0) and (1.1) and the example is complete.

With polynomials of higher degree the existence of more periodic solutions can be established. (The argument of Serbin fails in that he does not recognize the restricted range of the monotonicity of $\phi$, in Lefschetz's notation, as stated by Lefschetz [1, p. 193] and to which Serbin refers.)

\section{REFERENCES}

1. S. Lefschetz, Lectures on differential equations, Princeton, 1946.

2. N. Levinson and O. K. Smith, A general equation for relaxation oscillations, Duke Math. Journal 9, 382-403 (1942).

3. A. Liénard, Étude des oscillations entretenues, Rev. Gen. d'Élect. 23, 901-946 (1928).

4. H. Poincaré. Les méthodes nouvelles de la mécanique céleste, Vol. 1, Gauthier-Villars, Paris, 1892, Chap. III.

5. H. Serbin, Periodic motions of a nonlinear dynamic system, Q. Appl. Math. 8, 296-303 (1950).

\section{THE COMPRESSIBLE FLOW CORRESPONDING TO A LINE DOUBLET*}

\section{By J. W. CRAGGS (St. John's College, Cambridge)}

Introduction. The use of the hodograph equations for irrotational compressible flow in two dimensions leads, as noticed by Chaplygin ${ }^{1}$ and others, to a method of constructing compressible flow patterns by reference to similar problems in incompressible flow. For certain elementary flows, as for example the flow due to a line source, a line

${ }^{*}$ Received December 11, 1950.

${ }^{1}$ Chaplygin, On gaseous jets, Ann. Sci. Moscow Imperial University, Math.-Phys. section 21, (1904). 